\title{
Impuesto a la riqueza en épocas de la COVID-19: El caso peruano
}

\author{
Wealth Tax in Times of COVID-19: The Peruvian case
}

\author{
Renzo Vidal Caychoํㅜ, Julio Yarasca $\mathrm{Moscol}^{2}$
}

\begin{abstract}
The government of Peru has elaborated an economic policy to contain the economic disaster caused by COVID-19. In order to quickly revive the peruvian economy, the government adopts some measures in short term, and others are still under evaluation, such as imposition of a wealth tax or called as "solidarity tax" on people with higher annual incomes in order to increase the tax collection and thus sustaining public spending in coming quarters. With an unemployment level that marks a record $25 \%$ between February and April 2020, how does this measure impact to the consumption of peruvians? The effect is analyzed, showing the peruvian consumer profile and how their marginal propensity to consume could change with variation in their incomes.
\end{abstract}

Keywords: propensity marginal of consumption; taxes; discret regression

\section{RESUMEN}

La política económica del gobierno peruano es llegar a contener el desastre económico ocasionado por la COVID-19. Con la finalidad de reactivar rápidamente la economía, el gobierno adopta algunas medidas y otras aún continúan en evaluación, como por ejemplo, la imposición de un tributo a las riquezas o llamado "impuesto solidario" a las personas con mayores ingresos anuales que permita mayor recaudación tributaria y así sostener el gasto público en los próximos trimestres. Con un nivel de desempleo que marca un récord de $25 \%$ entre febrero y abril del 2020, ¿cómo impacta dicha medida al consumo de los peruanos?. Se analiza cuál es el efecto a dicha población mostrándose para ello el perfil del poblador peruano y qué tan susceptible podría ser su propensión marginal al consumir tras la variación de sus ingresos.

Palabras clave: propensión marginal a consumir; tributo; regresión discreta

Received: 18 de junio del 2020

Accepted: 5 de setiembre de 2020

\section{Introducción}

El presente artículo analiza la propuesta del gobierno peruano en implementar un impuesto a la riqueza al estrato socioeconómico con más altos ingresos, tras la considerable contracción de la recaudación tributaria post COVID-19. Según datos de la Superintendencia Nacional de Administración Tributaria (SUNAT), del 2010 al 2019 la presión tributaria promedio se ubica en $16 \%$ como porcentaje del PBI. Sólo en los primeros cuatro meses la recaudación tributaria presenta una caída abrumadora de $15 \%$ respecto al mismo período del 2019 , por lo que se considera la posibilidad de que la presión tributaria retroceda a $13 \%$ del $\mathrm{PBI}^{*}$ o una cifra menor, supeditado a la duración del aislamiento social y a la paralización de diversos sectores económicos.

Así, analizar cómo impactan los impuestos sobre el consumo es de vital importancia para conocer con mayor detalle la controversia entre crecimiento económico y desigualdad. Por ejemplo, Forbes (2000), tomando como muestra 40 países, sugiere que en el corto y mediano plazo el incremento en el nivel de desigualdad en los ingresos tiene una subsecuente relación positiva y significativa con el crecimiento económico. En cambio, Chang y Ram (2000), tomando datos de países de África, Europa y Latinoamérica, encuentran que el crecimiento económico sólo se generaría tras una menor desigualdad.

Al introducir en el análisis los impuestos, Myles (2009); Delgado y Salinas (2010) infieren que un mayor crecimiento económico depende de la transición de los países a impuestos indirectos, es decir a un incremento de la base tributaria en las imposiciones al consumo (IGV o IVA). Mientras que Jaramillo y Sparrow (2013) demuestran que si existe una reducción de impuestos en sociedades vulnerables, éstas apenas reducirían la desigualdad y peor aún, tendrían un impacto nulo al introducirse la informalidad en la muestra.

Entonces, ¿deberían incrementarse los impuestos a la sociedad en su conjunto?, como consecuencia no sólo ¿generarían un mayor crecimiento económico?, sino que también ¿no afectaría a la desigualdad?. En el reciente estudio de Piketty et al. (2018) muestran que en las últimas décadas diversos países vienen generando cada vez menos impuestos progresivos, que aunado a que algunos países emergentes aún continúan careciendo de sistemas de recaudación y de transferencia poco desarrolladas, reforzaría posiblemente el argumento de una mayor desigualdad en

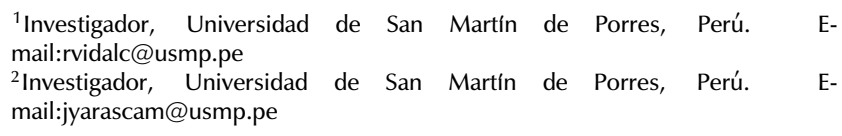

How to cite: Vidal Caycho, R., \& Yarasca Moscol, J. E. (2021). Impuesto a la riqueza en épocas de la COVID-19: El caso peruano. ANÁLISIS ECONÓMICO Y FINANCIERO, 4(1), 31-39. https://doi.org/10.24265/raef.2021.v4n1.32

\section{c) (1) (2)}

No Comercial-Compartir Igual 4.0 Internacional.

*Según el Global Economic Prospect del Banco Mundial (2020), el crecimiento económico del Perú para el 2020 es de $-12 \%$ respecto al año añterior.

${ }^{\dagger}$ Los impuestos progresivos se definen como el incremento de la tasa impositiva a aquellos contribuyentes que generan mayores ingresos (p.e. el impuesto a la renta). 
dichas sociedades e incrementaría la brecha entre ingresos y riquezas de las personas.

Entre las posiciones sobre la creación de tributos a la riqueza, se encuentra un estudio reciente pre COVID-19 de Saez y Zucman (2019), quienes fundamentan diversos factores, entre ellos, la capacidad de ser un mecanismo poderoso para restaurar el impuesto progresivo que permita generar mayor recaudación y desconcentrar la riqueza. Sólo en EEUU, las primeras 400 personas consideradas multimillonarias pagan relativamente menos que la clase media. El siguiente factor es el fortalecimiento de la institucionalidad en los sistemas de recaudación, evidenciándose pobres manejos de una política tributaria incapaz de generar riqueza al Estado y una sociedad más igualitaria. Mientras que el último factor es la elección enérgica de dicha política pública como forma de gobernar a la sociedad.

Por lo tanto, dilucidar si es favorable o no el impacto que podría tener el establecimiento de un tributo a la riqueza a las personas con mayor ingreso toma una particular relevancia económica, permitiendo generar una mejor evaluación sobre la política tributaria en épocas de la COVID-19, ya que la economía peruana posiblemente muestre una fuerte recesión en los próximos trimestres producida por la crisis sanitaria, lo que deterioraría el sistema tributario afectado por una menor recaudación en los siguientes años.

Así, el presente documento no tiene como objetivo tomar posición por alguno de los puntos de vista señalados, sino que debería analizarse de manera objetiva si conviene al Estado generar este tipo de impuestos a aquellas personas que generan mayores ganancias anuales. Para demostrarlo, se utilizan los datos proporcionados en la Encuesta Nacional de Hogares 2018 (ENAHO) del Instituto Nacional de Estadística e Informática, aplicando para ello modelos de probabilidad lineal y logística con la finalidad. Así, en la sección 2 se desarrolla la motivación y la importancia de este estudio; en la sección 3 se presenta una breve revisión de la literatura sobre política fiscal y desigualdad; en la sección 4 se proporciona el modelo teórico a implementar; y por último en la sección 5 y 6 se brindan los resultados y conclusiones obtenidas.

\section{Motivación}

Una de los principales retos que asume el gobierno peruano es tener una política económica de corto plazo capaz de generar aquel impacto que permita reactivar rápidamente a la economía peruana. Sin embargo, tras lanzar el programa "Reactiva Perú" con cerca del $12.3 \%$ del PBI, el gobierno propuso evaluar otras medidas que motiva un análisis más profundo, como aquella donde se propone imponer un tributo a las riquezas a las personas con ingresos altos, con la finalidad de generar mayores recursos públicos al fisco, permitiendo sostener el actual gasto púlico que se viene dando tras el estado de emergencia.

¿Es aquella decisión gubernamental una buena medida? ¿Cuánto impactaría en los hogares peruanos una medida de este tipo?. Estudios como el realizado por Benítez y Velayos (2017) muestran un breve diagnóstico sobre el impuesto a las riquezas, observando que las recaudaciones por dicho impuesto son muy poco significativas según las evidencias mostradas por distintos países, salvo Suiza que muestra cierta significancia (1\% del PBI en el 2015) (ver Tabla 1). En principio, dicho estudio no presenta un impuesto mínimo para evaluar, sugiriendo que el gobierno pueda fijarlo sin mayores estudios técnicos rigurosos que exige tener este tipo de medidas.

Desde el punto de vista de la teoría económica, la función consumo tiene varios matices y profundos pensamientos ideológicos que permite observarla desde varios ángulos. Uno de ello, es la función consumo propuesta por J. M. Keynes $^{\S}$, quien modela de manera muy sencilla que el consumo $\left(C_{i}\right)$ en el corto plazo depende de un consumo autónomo $\left(c_{0}\right)$ el cual se gasta en cada cierto período, independientemente de las condiciones económicas; y del ingreso disponble $\left(Y d_{i}\right)$ que es la diferencia entre el ingreso $\left(Y_{i}\right)$ que perciben las personas después de pagar impuestos $\left(T_{i}\right)$ (ver Ecuación 1).

$$
C_{i}=c_{0}+c_{Y d_{i}} *\left(Y_{i}-T_{i}\right)
$$

Así, un incremento de los impuestos al ingreso (impuesto a la renta) afecta de manera negativa a los ingresos disponibles y como consecuencia disminuye el consumo. Bajo la evidencia de De Cesare y Lazo (2008) quienes correlacionan los ingresos provenientes de los impuestos al patrimonio y el Coeficiente de Gini ${ }^{\pi}$, se observa que a medida que el gobierno coloca un impuesto al patrimonio, el resultado es una mayor desigualdad en dichos países(ver Figura 1).

Sin embargo, dentro de dicha ecuación sencilla existe un parámetro que muchos asumen que se encuentra inalterada en el tiempo: la Propensión Marginal a Consumir (PMgC), la cual se define como la proporción del ingreso que va directo al consumo. Tomando la Encuesta Nacional de Hogares (ENAHO) generada por el Instituto Nacional de Estadística e Informática (INEI), se construye dicho parámetro a partir de la división entre el gasto bruto total anual y el ingreso bruto total anual; clasificándolo entre "Pobres" y "No Pobres" de la muestra representativa. Del mismo modo, tomando en consideración a la teoría económica, existe también otro parámetro que define la proporción del ingreso que va directo al ahorro, llamada la Propensión Marginal a Ahorrar (PMgA). Así, la suma de ambas definiria las proporciones que tiene el individuo tanto para consumir como para ahorrar en función al ingreso que dispone (ver Ecuación 2).

$$
P M g C_{i}+P M g A_{i}=1
$$

Al dividir a la sociedad en dos grandes frentes como "No Pobres" y "Pobres", estando en este último grupo los pobres extremos, se analizan a través de diversos ploteos la $\mathrm{PMgC}$ respecto a los percentiles del ingreso (definiéndose al Percentil 1 como aquellos individuos que perciben los ingresos más bajos de la muestra; y el Percentil 100 cuyos

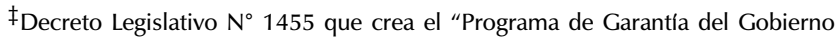
Nacional para la Continuidad en la Cadena de Pagos" (REACTIVA PERÚ), que tiene por objeto garantizar el financiamiento de la reposición de los fondos de capital de trabajo de empresas que enfrentan pagos y obligaciones de corto plazo con sus trabajadores y proveedores de bienes y servicios, a través de un mecanismo que otorgue la garantía del Gobierno Nacional a los créditos en moneda nacional que sean colocados por las Empresas del Sistema Financiero (ESF).

\$Teoría desarrollada en el libro The General Theory of Employment, Interest and Money (1936).

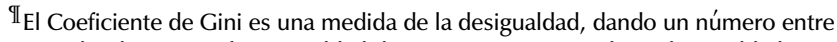
0 y 1 , donde 0 es perfecta igualdad de ingresos; y 1 es perfecta desigualdad (una persona acumula los ingresos de un país). En cambio, el índice de Gini es el coeficiente de Gini expresado en referencia a 100. 
de ingresos se encuentran entre lo más alto de la población representativa) (ver Figura 23 4). La principal idea es conocer cómo se comportan los diferentes consumos de los individuos que se encuentran en los diferentes percentiles, observándose que un grupo del sector socioeconómico considerado "Pobre" destinan gran parte de sus ingresos al consumo $0.9 \%$ y este va descendiendo hasta llegar a otro grupo que destina $0.2 \%$ de sus ingresos al consumo. Es decir, a medida que el nivel de ingreso se incrementa, la $\mathrm{PMgC}$ disminuye. De la misma manera, la PMgC del sector socioeconómico considerado "No Pobre", la $\mathrm{PMgC}$ se decanta entre $0.8 \%$ y $0.5 \%$, respectivamente. Al compararlas, los "Pobres" tienen una PMgC menos estrecho lo que puede considerarse que sus hábitos de consumo podrían cambiar rápidamente en función a los ingresos generados; mientras que los "No Pobres" tienen un hábito de consumo más estrecho y por lo tanto más inalterado.

Entonces, se podría pensar que al incrementar los impuestos a la renta, el consumo descendería en la misma proporción que descendería el ingreso. Pero la evidencia demuestra lo contrario, ya que la PMgC cambia a medida que los ingresos suben o caen. Por lo tanto, la PMgC está muy relacionado a la fuerza de los hábitos de consumo en las familias que no evolucionan de la misma manera que estas variables, sino que dichas costumbres en el consumo se mantienen en el tiempo, alterando por consiguiente la $\mathrm{PMgC}$ de las personas (Bejarano y Vidal, 2018).

Asimismo, se pensaría que los ingresos y por tanto el consumo son algo estables en el tiempo, pero ello depende de la evolución de las economías; y más aún por la capacidad de generar nuevos ingresos (trabajos secundarios) y hasta el hecho de almacenar alimentos que permita generar un consumo controlado. En ese sentido, desde el lado de los ingresos según los datos de la ENAHO (INEI), la principal ocupación de un ciudadano peruano es trabajador independiente (39\%), dos veces más que ser empleado (19\%) u obrero (18\%) (ver Figura 5). Mientras que la frecuencia con que le pagan al trabajador es diaria (1\% del total); semanal (26\%); quincenal (6\%); y mensual (67\%); ganando en promedio S/. 38 soles diarios; S/. 202 soles semanales; S/. 555 soles quincenales; y S/. 1,719 soles, respectivamente.

Mientras que el análisis de un mejor gasto de consumo controlado, que puede estar relacionado al hecho de almacenar alimentos, la proporción de peruanos que cuentan con una refrigeradora en Lima es aproximadamente del $24 \%$ de la población, muy por encima de las demás regiones cuya cifra promedio es extremadamente más baja con apenas un $3.2 \%$ de la población que cuenta con dicho aparato.

Por lo tanto, la pregunta central que pretender resolver el presente trabajo es si al aumentar los impuestos a la población con mayores ingresos ¿qué tan probable es que retroceda su consumo tras los menores ingresos?. Según la Figura 3, el efecto del impuesto sobre la PMgC es indirecto, por lo que se esperaría un incremento poco considerable de la PMgC y consecuentemente una mínima caída en el consumo, considerado como el principal motor de la economíall.

\section{Breve reseña literaria}

En esta sección se analizará cómo la generación de una política fiscal en base a una mayor tributación tiene un impacto principalmente de países emergentes, lo que podría generar una mayor desigualdad e inequidad en la sociedad. Asimismo, la mayor recaudación de tributos que posiblemente se generaría a partir de tasas escalonadas, se ve diezmada por la poca efectividad y la informalidad en varios países.

El análisis de la política fiscal se basa en el buen funcionamiento del sistema tributario para que el gasto público alcance la máxima eficiencia y equidad (Tanzi y Zee, 2000). El rol de la política fiscal en los países en vías de desarrollo resulta ser un tema sensible porque su sistema tributario tiene que recaudar eficientemente sin endeudarse, tratar de no desincentivar la actividad económica y que al ser un país emergente, no se aleje de las normas internacionales.

Los países emergentes presentan frecuentemente estructuras tributarias ineficientes debido a cierta precariedad de sus actividades económicas y también a dificultades fiscales que amenazan una reforma integral de los impuestos.

De acuerdo con Tanzi y Zee (2000) los países emergentes con frecuencia muestran desigualdad respecto de los ingresos teniendo un coeficiente de Gini mayor al 0.5. La explicación de esta desigualdad implica que para aumentar los ingresos tributarios habría que gravar a los que reciben mayores ingresos, pero se encuentra la dificultad de que los que perciben mayores ingresos son los que se encuentran dentro del poder económico y político frenando toda reforma fiscal.

Si bien la imposición de un impuesto interfiere en la pérdida de bienestar al distorsionar las decisiones de los agentes económicos, resulta importante realizar un análisis respecto al impuesto a los ingresos o impuesto a la renta.

El impuesto a la renta en los países en vías de desarrollo según Barreix, Benítez y Pecho (2017) afirman que se trata de un impuesto progresivo y que mayormente es pagado por un pequeño grupo formalizado que reciben altos ingresos. El impuesto a la renta lo paga el $80 \%$ del decil más rico, aunque sus tasas efectivas son muy bajas por lo cual no se encuentra una verdadera redistribución del ingreso en los países en vías de desarrollo.

Como se ha visto anteriormente para lograr que el impuesto a la renta tenga el impacto necesario en la redistribución del ingreso tendría que aumentar las tasas efectivas, pero esto a su vez resulta controversial porque los individuos con ingresos altos son los que aportan más a la acumulación de capital e innovación (Serra, 2006). Al concentrarse el ingreso en una minoría genera más desigualdad en la sociedad. Generalmente los países en desarrollo cuentan con una mayor recaudación tributaria en los impuestos indirectos y los impuestos a la explotación de los recursos naturales, desincentivando ampliar la base tributaria sobre todo en el impuesto a los ingresos o impuesto a la renta.

Por lo tanto, resulta necesario analizar y contestar lo siguiente: ¿cuál sería el impacto al imponer un nuevo impuesto a la renta?; ¿contribuiría al bienestar social una reforma del impuesto a la renta?; ¿las personas de más altos ingresos deben pagar más impuestos? Para dar

\footnotetext{
"Ver capítulo VII del libro Advanced Macroeconomics (2006) de David Romer.
} 
respuesta a estas preguntas primero debe analizarse que pasaría en una economía emergente y después se trasladaría a algunos casos específicos de países latinoamericanos y que resultados mostraron al analizar la eficiencia del impuesto a la renta.

La importancia del impuesto a la renta está fuera de discusión, sobre todo porque ha sido muy relevante en los países desarrollados donde es el impuesto que recauda más**. Resulta importante señalar que cuenta con un potencial redistributivo importante a comparación con su eficiencia. Al ser un impuesto progresivo su objetivo es lograr la equidad, factor importante que no se encuentra en los países emergentes (Barreix, Benítez y Pecho, 2017).

Un punto importante para señalar es que los países emergentes se enfocan por mantener varios tramos del impuesto a la renta con tasas nominales altas para favorecer sus posturas políticas de justicia social, aunque en la práctica exista múltiples exenciones o evasiones al impuesto. Inclusive observando varias experiencias de reformas del impuesto a la renta se puede llegar a analizar que la progresividad de las tasas efectivas de este impuesto se vería favorecido si se redujera el grado de progresividad de las tasas nominales y el número de tramos, también que se redujeran las exenciones y deducciones (Tanzi y Zee, 2000).

El impuesto a la renta por naturaleza es progresivo, esto quiere decir que las personas con más altos ingresos pagan una proporción mayor de impuestos. Sin embargo, muy pocas personas pagan las tasas efectivas promedio en comparación con una tasa legal mínima promedio, por esto es que los objetivos de aumentar la recaudación tributaria y como consecuencia la redistribución, se ven efectivamente mermadas.

Si bien el impuesto a la riqueza en muchos países emergentes es progresivo, tiene aún un ínfimo poder redistribuidor. A raíz de esto, según el trabajo de Barreix, Benítez y Pecho (2017) al utilizar el índice ReynoldsSmolensky, que mide la diferencia entre la distribución del ingreso antes y después del impuesto a la renta y que cuantifica el grado de inequidad que se ha reducido, observan que el índice para los países de América Latina es muy bajo confirmando la poca capacidad redistributiva de la región.

Analizando a Ecuador que es un caso particular y similar al Perú, según Ramírez y Carrillo (2017) que utilizan como base la metodología del balance estructural para medir las elasticidades y las eficiencias del impuesto a la renta respecto al $\mathrm{PBI}$, encuentran que la eficiencia en la recaudación se ha logrado gracias a una reforma de la administración tributaria. A raíz de este estudio se observa que la eficiencia del impuesto a la renta tiene un crecimiento sostenido en un determinado período esto como consecuencia de un eficiente funcionamiento de la administración tributaria.

En conclusión, el impuesto a la renta resulta ser importante en los países desarrollados en contraste con los países emergentes. El impuesto a la renta al ser un impuesto progresivo y que resulta en una herramienta importante para reducir la desigualdad es ineficiente en los paises emergentes por las múltiples exenciones o distorsiones que presenta. También esta ineficiencia tiene que ver con la poca importancia y un sistema tributario deficiente en estos países, como por las tasas efectivas que se imponen. Por lo tanto, para que resulte eficiente un impuesto a la renta en dichos países se tendría que mejorar la institucionalidad del sistema tributario y realizar una reforma profunda a determinado impuesto para que su capacidad redistributiva genere el bienestar deseado a la sociedad.

\section{Metodología}

En esta sección se presenta un modelo sencillo de no linealidad que permita conocer la probabilidad de que las personas puedan ser más pobres al aumentarle los impuestos.

\section{Modelo de probabilidad lineal (MPL)}

Primeramente se presenta el modelo de probabilidad lineal (MPL) que permita realizar una comparación con los modelos no lineales. El MPL se define de manera muy simple que es la relación de una variable dependiente $Y_{i}$ con una variable independiente $X_{i}$ (ver Ecuación 3).

$$
Y_{i}=X_{i}^{\prime} \beta+\xi_{i}
$$

Asĺ, los valores a lo largo de la recta estimada nos indican la probabilidad asociada a la ocurrencia o no del evento estudiado $(\hat{Y})$. En este modelo, dado que la variable dependiente es cualitativa, la expectativa condicional es $E(Y i \mid X i)$ interpretándose como la probabilidad condicional de que ocurra el evento, es decir, definiéndose como $\operatorname{Pr}(Y i=1 \mid X i)$.

De esta manera, los coeficientes estimados mediante el MPL serían los efectos marginales o cambios en la probabilidad de que ocurra este evento. ¿Por qué se puede aplicar un modelo de Mínimos Cuadrados Ordinarios (MCO)? Esto porque si uno toma en consideración que la dependiente es una variable que indica ocurrencia o no de un evento, entonces seguiría una distribución de Bernoulli, por ende su función de distribución sería: $f\left(Y_{i}\right)=\operatorname{Pr}_{i}^{Y_{i}} *\left(1-P r_{i}\right)^{\left(1-Y_{i}\right)}$.

Entonces, la probabilidad de que el evento ocurra es $Y_{i}=1$ que es igual a $P_{i}$ y la otra probabilidad de que no ocurra es $Y_{i}=0$ que es igual a $1-P_{i}$. Así, la ecuación final sería (ver Ecuación 4) la expectativa condicional en función a las variables independientes, donde los errores de la ecuación no seguirian una distribución normal sino una distribución Bernoulli como antes se mencionó.

$$
E\left(Y_{i} / X_{i}\right)=\beta_{0}+X_{i} \beta_{1}=P_{i}
$$

Sin embargo, el utilizar un MCO conllevaría a estimar errores que son no normales debido a la variable bivariada dependiente, obteniéndose una varianza no constante y errores heterocedásticos. Por lo tanto, se debe ampliar el análisis con la utilización de modelos no lineales.

\section{Modelo no lineales o de elección discreta}

Los modelos no lineales se utilizan cuando la variable dependiente es cualitativa (atributo), donde estos tipos de

\footnotetext{
**Según Arias (2011) dentro de la estructura de recaudación peruana, los impuestos indirectos es mayor al de los impuestos directos lo que genera un menor impacto en la equidad de la población
} 
modelos dependen de la distrubución que se asuma para la función enlace. Asimismo, estos modelos cuentan con funciones diferenciables.

En lo sucesivo se realiza un modelo de utilidad aleatoria, cuyo enfoque debe adoptar una decisión que le permita elegir entre dos alternativas (Pobre o No Pobre), la cual maximize su utilidad esperada que le proporciona cada una de estas alternativas. Entonces el modelo probabilístico estaría dado por una variable entre 1 y 0 (ver Ecuaciones 5 y 6), donde si la variable binaria $Y_{i}=1$, entonces la $U_{i, 1}>U_{i, 0} ;$ mientras que si $Y_{i}=0$, entonces la $U_{i, 1}<U_{i, 0}$.

$$
\begin{aligned}
& U_{i, 0}=a_{0}+X_{i, 0} \beta+\xi_{i, 0} \\
& U_{i, 1}=a_{1}+X_{i, 1} \beta+\xi_{i, 1}
\end{aligned}
$$

Por lo tanto, el modelo probabilístico sería la siguiente Ecuación 7:

$$
\operatorname{Pr}\left(Y_{1}=1\right)=\operatorname{Pr}\left(U_{i, 1}>U_{i, 0}\right)=\operatorname{Pr}\left(U_{i, 1}-U_{i, 0}>0\right)=F\left(X_{i} b\right)
$$

Bajo estos argumentos, se elije utilizar dos modelos que permitan resolver la pregunta central de la investigación que es si al aumentar los impuestos a la población, ¿qué tan probable es que se vuelvan pobres?. En ese sentido, realizaremos dos modelos de regresión no lineal, siendo el modelo Logit y el modelo Probit. Ambos modelos permiten acotar la predicción dado que se modela la probabilidad de que ocurra el evento. Asimismo, el resultado al aplicar dichos modelos no es interpretar dicho $\beta$ sino que se deben hallar los efectos marginales, es decir en cuanto cambia la variable dependiente ante un cambio en una unidad de la variable independiente.

La diferencia entre ambos modelos Logit y Probit se encuentra en la función de enlace para la parametrización del modelo ${ }^{\S \S}$. Así, se tiene que la función logística es no lineal en las $X_{i}$ ni en los coeficientes $\beta_{i}$ (ver Ecuaciones 8 y 9).

$$
\begin{gathered}
\operatorname{Pr}_{i}=\frac{e^{-X_{i}^{\prime} \beta}}{1+e^{X_{i}^{\prime} \beta}} \\
1-P r_{i}=\frac{1}{1+e^{X_{i}^{\prime} \beta}}
\end{gathered}
$$

Por lo tanto, a partir de estas ecuaciones se puede obtener la razón de probabilidades ("Odds Ratio") de que ocurra o no ocurra el evento lo que permite tener una mejor inferencia del modelo (ver Ecuación 10), además de las estimaciones de los $\beta_{i}$ aplicando el logaritmo neperiano a dicha razón de probabilidades (ver Ecuación 11).

$$
\begin{gathered}
\frac{P r_{i}}{1-P r_{i}}=e^{X_{i}^{\prime} \beta} \\
L_{i}=\operatorname{Ln}\left(\frac{P r_{i}}{1-P r_{i}}\right)=X_{i}^{\prime} \beta
\end{gathered}
$$

\section{Modelo propuesto}

Para resolver la pregunta central, se realiza el modelo Logit y Probit. Para ello se utiliza la base de datos que proporciona la ENAHO (INEI) del año 2018, obteniéndose los percentiles por ingreso ${ }^{\text {ॠा }}$ para la construcción de la variable dependiente binaria o dicotómicas. Esta se divide en aquellos ingresos por debajo del percentil 30 que se consideran igual a cero (0) considerada "Pobre" y aquellas cuyos ingresos están por encima del percentil 30 considerándose igual a uno (1) considerada "No Pobre". Del mismo modo, como variables independientes se consideran tres (3) grupos de variables que determinan las características del individuo, si se encuentran en la condición socioeconómica "Pobre" o "No Pobre".

Para ello, se considera un primer grupo son las características propias de la persona encuestada como su edad, sexo, DNI, sabe leer y escribir, condición de pobreza; además del estado conyugal y su relación con el jefe del hogar. Un segundo grupo son las condiciones de la vivienda como si es propia o alquilada; además de los sumnistros de servicios básicos como la forma que llega el agua a la vivienda, si es potable; además del uso de la energía eléctrica; y el uso de las comunicaciones como el teléfono, internet, cable o ningún servicio. Un tercer grupo se encuentran temas de salud y previsiones como si el encuestrado o algún miembro de la familia presenta enfermedades recurrentes así como la afiliación al sistema nacional previsional (privado - AFP o público - ONP).

Como se detalla al inicio del trabajo, un incremento en el número de impuestos a la riqueza ocasiona una caída en el ingreso disponible $Y d$ y como consecuencia en el consumo. No obstante, existe una PMgC que no permite que ambas desciendan en la misma proporción ya que existen fuertes hábitos de consumo. Por lo tanto, se espera que dicho parámetro se incremente a medida que el $Y d$ disminuya. Entonces, ¿qué tan probable es que los ingresos de los consumidores peruanos, que se encuentran en los percentiles más altos de ingresos, retrocedan al no poder cambiar de hábitos de consumo reflejados en un incremento de la PMgC?.

\section{Principales resultados}

Realizando las estimaciones tanto del modelo Logit como del modelo Probit así como sus efectos marginales en aquella población cuyo percentil se encuentra por encima de la línea de pobreza, los cuales permiten observar el impacto de cada variable (ver Tabla 2), se puede analizar que se encuentran correctamente clasificados ( $83 \%$ cada una) con pruebas de significancia conjunta bastante aceptables (ver

\footnotetext{
${ }^{\dagger+}$ monótona creciente con dominio en los números reales $(R)$ y un rango entre 0 y 1.

††Para ello, se utiliza el modelo de Máxima Verosimilitud (MV) el cual permite obtener coeficientes $\beta_{i}$ que maximizan la función de verosimilitud y que mejor se ajustan a los datos. La estimación de dichos parámetros se da maximizando la variable $L=f\left(\beta ; Y_{i}, \ldots Y_{n} \mid X_{i}, \ldots X_{n}\right)=\prod_{i=1}^{n}\left[\varphi\left(X_{i} \beta\right)^{Y_{i}}+\left(1-\varphi\left(X_{i} \beta\right)\right)^{1-Y_{i}}\right]$. Luego aplicando logaritmos y derivando respecto a $\beta$, se obtienen los $\hat{\beta}$. Asimismo, en muestras grandes, el MV es consistente, se distribuye de manera uniforme y es eficiente.

$\S \S$ La función de distribución de un modelo Logit sigue una función logística; mientras que la función de distribución de un modelo Probit sigue una distribución normal, respectivamente: $\left(\mathrm{P}_{i}=\frac{1}{\sqrt{2 \pi}} \int_{-\infty}^{X_{i}^{\prime}} e^{\frac{-Z^{2}}{2}} d Z\right) y\left(P_{i}=\frac{1}{1+e^{-X_{i}^{\prime} \beta}}\right)$.

III Construido a partir de los ingresos brutos totales anuales de la muestra.
} 
Tabla 3). Asimismo, se puede verificar que la PMgC es una de las variables con mayor explicación del modelo logístico. En tanto, el impacto que tiene la $\mathrm{PMgC}$ sobre la variable binaria que refleja la condición de ser "No Pobre" o "Pobre" es indirecto, con un porcentaje de $32 \%$ y $34 \%$ respectivamente. Es decir, a medida que la $\mathrm{PMgC}$ se eleva, existe la probabilidad de que el consumidor pase de una condición de "No Pobre" a "Pobre".

Sin embargo, al observar las densidades de Kernel, el cual permite conocer la distribución de los intervalos de los valores que toma la probabilidad de ocurrencia del evento, son asimétricas a la izquierda y giran en torno a 1 (ver Figura 6 y 7) lo que se presume que hay altas probabilidades de continuar siendo un consumidor del sector socioeconómico "No Pobre" pese a cualquier posibilidad en el cambio de cualquier variable diagnosticada en el modelo. Asimismo, al realizar un ploteo de las probabilidades de ocurrencia respecto a la PMgC (ver Figura 89 y 10), se observa que a medida que la PMgC se eleva, la probabilidad de ocurrencia de que el consumidor sea "Pobre" es relativamente baja.

Por último, uno de los últimos indicadores que da respuesta a la pregunta central se encuentra en la Tabla de probabilidades de ocurrencia o el llamado "odds ratio" (ver Tabla 4). En dicha Tabla se puede observar que, dado que la PMgC tiene una relación indirecta con la variable binaria, a medida que la $\mathrm{PMgC}$ se incrementa en una unidad apenas es un $12 \%$ probable que el consumidor pase a la condición de "Pobre".

\section{Conclusiones}

Como se señala en el documento, la PMgC refleja la proporción del gasto de consumo sobre los ingresos de los ciudadanos. Este parámetro puede verse alterado fuertemente en función al incremento sustancial de los ingresos dentro de la categoría denominada "Pobres" o en aquellos percentiles más bajos de la cateogría denominada "No Pobres". Sin embargo, dentro de los percentiles más altos de esta última categoría, incrementos sustanciales en los ingresos no generaría mayor volatilidad de la $\mathrm{PMgC}$, por lo mismo que los hábitos de consumo son persistentes en el tiempo.

Por lo tanto, se puede concluir en base a los datos analizados en este documento, la posibilidad de establecer un impuesto a las riquezas en aquellos ciudadanos con los más altos ingresos anuales de la población y con PMgC muy bajas. Un pequeño ejercicio podría ser considerar a aquella población que presenta un ingreso anual por encima de $\mathrm{S} / .850,000$ soles con una PMgC de 0.4 , los cuales son aproximadamente el $1 \%$ de la muestra. El impacto no tendría efectos sobre su consumo dada la alta probabilidad de que su PMgC se mantenga invariable y sus ingresos no se vean afectados considerablemente. No obstante, la toma de posición de aplicar o no este impuesto en base a este documento, debe ser muy bien analizado por los hacedores de política tras los grandes efectos que podría ocasionar en la población, recomendándose que sea sólo bajo circunstancias temporales y de suma emergencia.

En lo que se refiere a los temas de agenda futura, se debería observar las condiciones de mercado donde se mueven estos ciudadanos con altos ingresos. La imposición de nuevos tributos a los sectores económicos, asumiendo que dicha población cuenta con grandes activos y conglomerados empresariales, cabría la posibilidad de que dichos impuestos sean trasladados al consumo de bienes y servicios finales, perjudicando la cantidad eficiente suministrada al mercado.

\section{Agradecimientos}

Los autores agradecen el apoyo del Instituto de Investigación de la Facultad de Ciencias Contables y Económicas y Financieras de la Universidad de San Martín de Porres.

\section{Referencias}

[1] Barreix, A., Benítez, J. C., Pecho, M.. 2017. Revisando el impuesto a la renta personal en América Latina: Evolución e impacto. CIAT. pp. 8.

[2] Bejarano, M., Vidal, R.. 2018. The impact of habits formation over social programs to reduce the poverty in Peru.. Revista de Análisis Económico y Financiero. Vol. 1, $N^{\circ} 1$, pp. 14.

[3] Benítez, J. Velayos, F.. 2017. Impuesto a la riqueza o al patrimonio de las personas físicas con especial mención en ALC. CIAT. pp. 84.

[4] Chang, J. Ram, R.. 2000. Level of Development, Rate of Economic Growth, and Income Inequality. Economic development and cultural change. Vol. 48, No. 4, pp. 787 - 799.

[5] De Cesare, C. Lazo, J.. 2008. Impuesto a los patrimonios en ALC. CEPAL. Serie Macroeconómica de Desarrollo. No. 126, pp. 41.

[6] Delgado, F. Salinas, J.. 2010. Impuesto y crecimiento económico: una panorámica. Revista Asturiana de Economía. No. 42, pp. 22.

[7] Forbes, K.. 2000. A reassessment of the relationship between inequality and growth.. American Economic Review. Vol. 90. No. 4, pp. 869 - 887.

[8] Jaramillo, M., Sparrow, B.. 2013. La incidencia del gasto social y los impuestos en el Perú.. GRADE. Documento de investigación. Pobreza y equidad. No. 70, pp. 56.

[9] JimÉnez, J. P., SolimANo, A.. 2012. Elites económicas, desigualdad y tributación.. CEPAL. Serie Macroeconómica de Desarrollo. No. 66, pp. 111.

[10] Johnson, C.. 2002. Una propuesta de reforma tributaria eficiente.. Estudios Públicos. No. 87, pp. 22.

[11] Myles, G.. 2009. EConomic growth and the role of taxation theory.. Economics Department, OCDE. Working Paper No. 713.

[12] Piketty, T., Saez, E. Zucman, G.. 2018. World inequality report 2018.. Harvard University Press.

[13] Ramírez, J., Carrillo Maldonado, P. A.. 2017. Indicador de eficiencia recaudatoria del impuesto al valor agregado y del impuesto a la renta de Ecuador. Documento de trabajo del BID. No. IDB-WP-87, pp. 26.

[14] SabainI, O., O'Farrell, J.. 2009. La economía política de la política tributaria en América Latina. 21 Seminario Regional de Política Fiscal / ILPES-CEPAL. Documento preliminar. 
[15] SerRa, P.. 2006. La reforma al sistema tributario chileno: una tarea inconclusa. Estudios Públicos. No. 101, pp. 26.

[16] Tanzı, V., Zee, H. H.. 2000. Tax policy for emerging markets: Developing countries.. IMF. WP/00/35.

\section{Figuras y tablas}

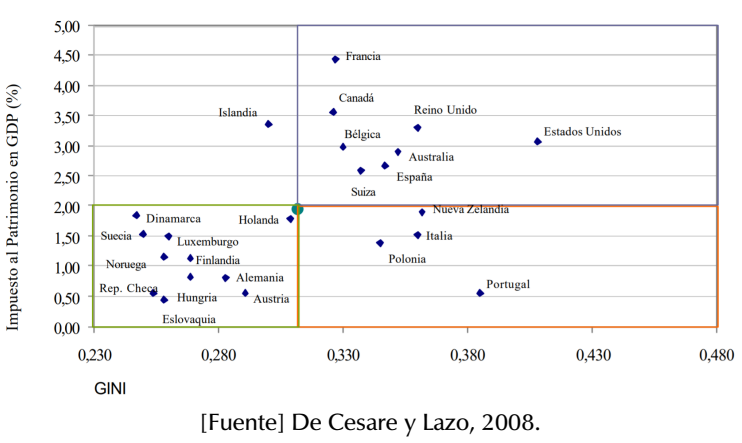

Figura 1. Impuestos al patrimonio vs. coeficiente de GINI, 2000 2004

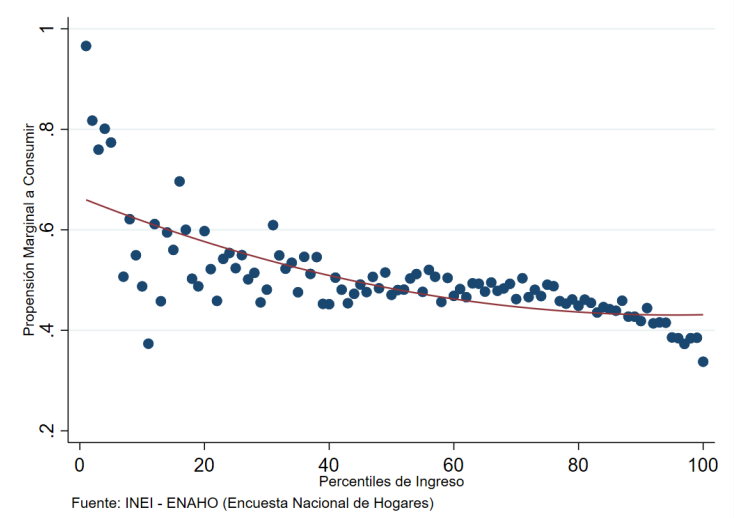

[Fuente] INEI - ENAHO.

Figura 2. PMgC por percentiles de ingreso disponible: No Pobres, 2018

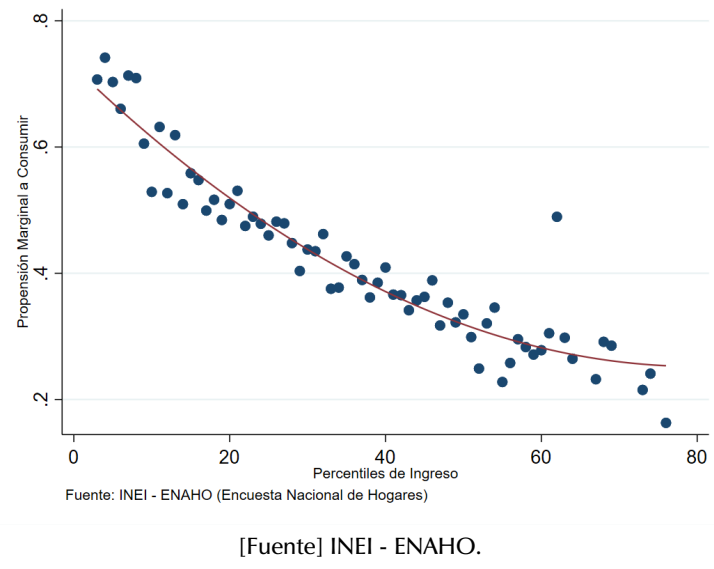

Figura 3. $\mathrm{PMgC}$ por percentiles de ingreso disponible: Pobres, 2018

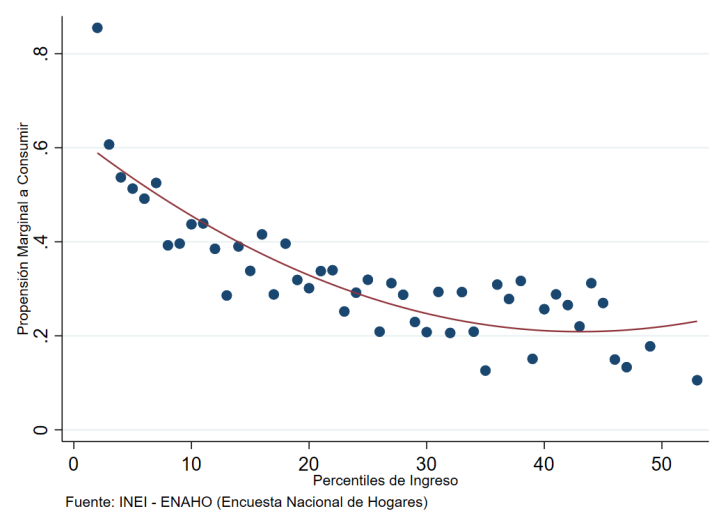

[Fuente] INEI - ENAHO.

Figura 4. $\mathrm{PMgC}$ por percentiles de ingreso disponible: Pobres extremos, 2018

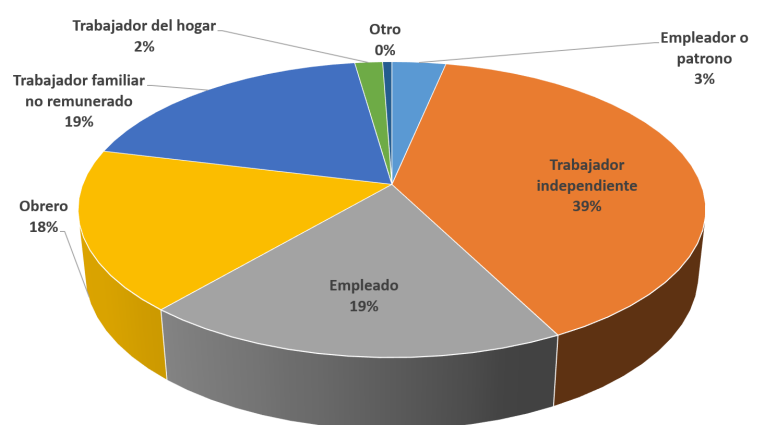

[Fuente] INEI - Enaho (Encuesta Nacional de Hogares). Elaboración propia.

Figura 5. Perú: Ocupación principal o negocio, 2018 


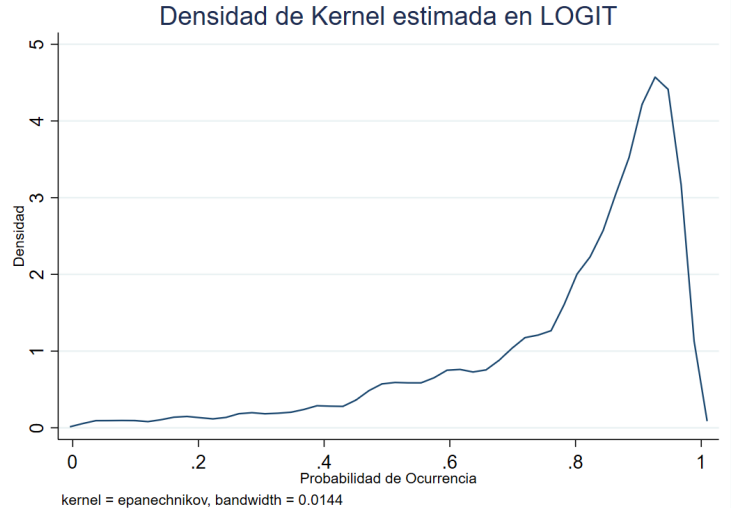

[Fuente] INEI - Enaho (Encuesta Nacional de Hogares). Elaboración propia.

Figura 6. Densidad de Kernel por modelo estimado: Logit

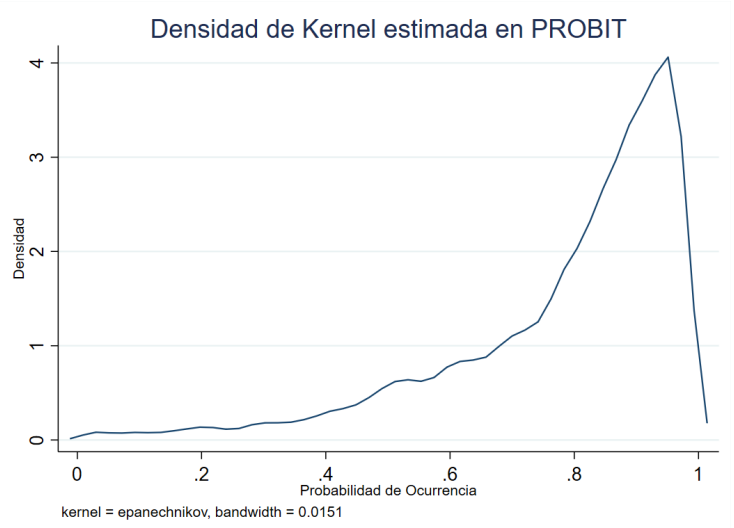

[Fuente] INEI - Enaho (Encuesta Nacional de Hogares). Elaboración propia.

Figura 7. Densidad de Kernel por modelo estimado: Probit

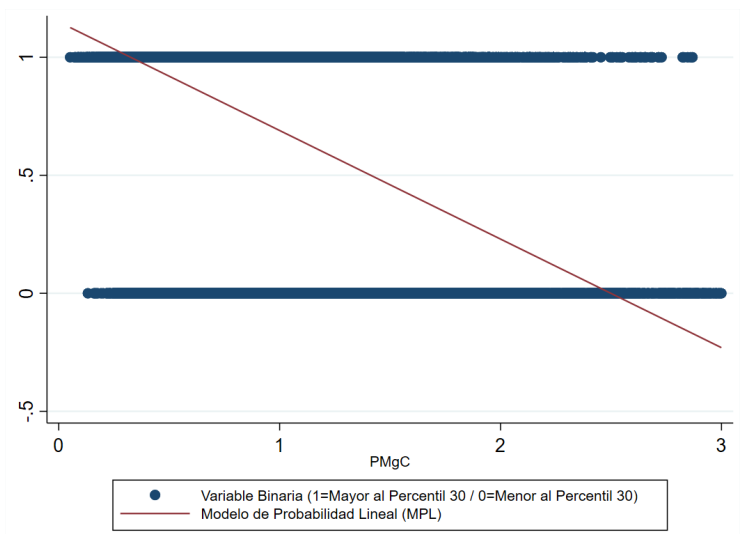

[Fuente] INEI - Enaho (Encuesta Nacional de Hogares). Elaboración propia.

Figura 8. Variable binaria vs. PMgC por modelo estimado: MPL

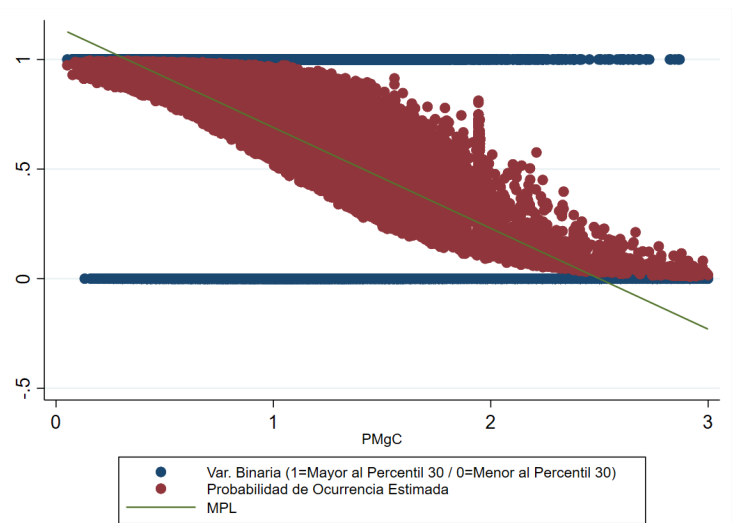

[Fuente] INEI - Enaho (Encuesta Nacional de Hogares). Elaboración propia.

Figura 9. Variable binaria vs. PMgC por modelo estimado: Logit

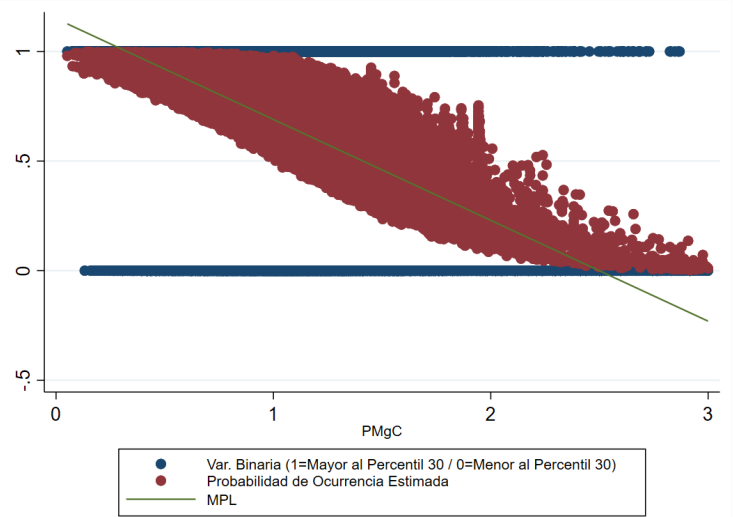

[Fuente] INEI - Enaho (Encuesta Nacional de Hogares). Elaboración propia.

Figura 10. Variable binaria vs. PMgC por modelo estimado: Probit

Tabla 1. Recaudación de impuestos a la propiedad y al patrimonio neto, $2014^{1}$

\begin{tabular}{lcccc}
\hline \multicolumn{1}{c}{ Región } & OCDE & ALC & África & Asia \\
\hline Al consumo de bienes y servicios & $11.0 \%$ & $11.2 \%$ & $11.3 \%$ & $5.6 \%$ \\
A los ingresos, ganancias y rentas & $11.6 \%$ & $6.2 \%$ & $7.2 \%$ & $7.7 \%$ \\
de capital & & & & \\
Sobre la propiedad inmueble & $1.1 \%$ & $.4 \%$ & $.3 \%$ & $.8 \%$ \\
A la riqueza & $.2 \%$ & $.1 \%$ & $.0 \%$ & $.0 \%$ \\
$\quad$ Recurrentes a la riqueza neta & $.2 \%$ & $.1 \%$ & $.0 \%$ & $.0 \%$ \\
$\quad$ No recurrentes a la riqueza neta & $.0 \%$ & $.0 \%$ & $.0 \%$ & $.0 \%$ \\
Recaudación tributaria total & $34.3 \%$ & $22.8 \%$ & $22.2 \%$ & $19.2 \%$ \\
\hline 1/ Son promedios para OCDE, LAC, África y Asia & & & &
\end{tabular}

[Fuente] OCDE (2017). Elaboración: Benítez y Velayos (2017). 
Tabla 2. Estimaciones con variable dependiente cualitativa

\begin{tabular}{|c|c|c|c|c|c|}
\hline & \multicolumn{3}{|c|}{ Estimaciones $^{1 / 2 /}$} & \multicolumn{2}{|c|}{ Efec. Marginal. } \\
\hline & MLP & Logit & Probit & Logit & Probit \\
\hline \multirow[t]{2}{*}{ Constante } & 1.43 & 4.87 & 3.28 & - & - \\
\hline & $(.016)$ & $(.077)$ & $(.077)$ & $(-)$ & $(-)$ \\
\hline \multirow[t]{2}{*}{$\mathrm{PMgC}$} & -.38 & -2.48 & -1.43 & -.32 & -.34 \\
\hline & $(.003)$ & $(.028)$ & $(.016)$ & (41.4) & $(.004)$ \\
\hline \multirow[t]{2}{*}{ Dominio } & -.01 & -.05 & -.02 & -.01 & -.01 \\
\hline & $(.000)$ & $(.004)$ & $(.002)$ & $(.001)$ & $(.001)$ \\
\hline \multirow[t]{2}{*}{ Vivienda } & .02 & .12 & .07 & .02 & .016 \\
\hline & $(.000)$ & (.008) & $(.004)$ & $(.001)$ & $(.001)$ \\
\hline \multirow[t]{2}{*}{ Agua Potable } & -.13 & -.92 & -.51 & -.12 & -.12 \\
\hline & $(.003)$ & $(.022)$ & $(.013)$ & $(.003)$ & $(.003)$ \\
\hline \multirow[t]{2}{*}{ Electricidad } & -.07 & -.40 & -.26 & -.05 & -.06 \\
\hline & $(.007)$ & $(.051)$ & $(.030)$ & $(.007)$ & $(.007)$ \\
\hline \multirow[t]{2}{*}{ Telefonía } & -.11 & .10 & -.42 & .014 & -.10 \\
\hline & $(.012)$ & $(.036)$ & $(.062)$ & $(.005)$ & $(.015)$ \\
\hline \multirow[t]{2}{*}{ Cable } & .03 & .25 & .12 & .03 & .03 \\
\hline & $(.003)$ & $(.023)$ & $(.013)$ & $(.003)$ & $(.003)$ \\
\hline \multirow[t]{2}{*}{ Internet } & .05 & .50 & .23 & .06 & .06 \\
\hline & $(.003)$ & $(.027)$ & $(.015)$ & $(.003)$ & $(.004)$ \\
\hline \multirow[t]{2}{*}{ Ningún Servic. } & -.14 & - & -.55 & - & -.13 \\
\hline & $(.014)$ & $(-)$ & $(.066)$ & $(-)$ & $(.016)$ \\
\hline \multirow[t]{2}{*}{ Jefe de Hogar } & .01 & .06 & .03 & .01 & .01 \\
\hline & $(.000)$ & $(.006)$ & $(.004)$ & $(.001)$ & $(.001)$ \\
\hline \multirow[t]{2}{*}{ Afiliación AFP } & .06 & .51 & .28 & .07 & .07 \\
\hline & $(.003)$ & $(.030)$ & $(.017)$ & $(.003)$ & $(.004)$ \\
\hline \multirow[t]{2}{*}{ Afiliación ONP } & .03 & - & .18 & - & .05 \\
\hline & $(.002)$ & $(-)$ & $(.010)$ & $(-)$ & $(.003)$ \\
\hline$R^{2}$ & 31.7 & 19.6 & 19.8 & 35.4 & 35.4 \\
\hline \multicolumn{6}{|c|}{$\begin{array}{l}\text { 1/ Todas las variables son significativas al 5\%.En paréntesis se encuentran lasdesviaciones estandar de } \\
\text { distribución Z. }\end{array}$} \\
\hline 2/ Bajo el R2 utilizad & cFadden. & & & & \\
\hline
\end{tabular}

Tabla 3. Clasificaciones estadísticas

\begin{tabular}{|c|c|c|}
\hline & Logit & Probit \\
\hline $\begin{array}{l}\text { Correctamente Clasi- } \\
\text { ficados }\end{array}$ & $82.8 \%$ & $82.7 \%$ \\
\hline $\begin{array}{l}\mathrm{R}^{2} \text { ajustado de } \\
\text { McFadden }^{1 /}\end{array}$ & $78.6 \%$ & $78.3 \%$ \\
\hline Especificidad & $28.3 \%$ & $27.0 \%$ \\
\hline Sensibilidad & $96.5 \%$ & $96.6 \%$ \\
\hline
\end{tabular}

$1 /$ Es el ratio entre el $N^{\circ}$ correctamente clasificados menos el $N^{\circ}$ de categoría

con más observaciones entre el $\mathrm{N}^{\circ}$ de obs. menos el $\mathrm{N}^{\circ}$ de categoría con más

observaciones

[Fuente] INEI - ENAHO (Encuesta Nacional de Hogares). Elaboración propia.

Tabla 4. Indicadores de ajuste del modelo estimado

\begin{tabular}{lcc}
\hline & Logit & Probit \\
\hline Chi - Cuadrado & & \\
Devianza & 63,009 & 61,241 \\
$\mathrm{P}$ - Value & $0.00 \%$ & $0.00 \%$ \\
\hline $\mathrm{R}^{2}$ & & \\
McFadden Ajustado & $19.6 \%$ & $19.9 \%$ \\
Cox - Snell & $17.8 \%$ & $18.1 \%$ \\
Cragg - Uhler / Nagelkerke & $28.2 \%$ & $28.5 \%$ \\
\hline Criterio de Información de Akaike & \\
AIC & 63,121 & 61,269 \\
BIC & 63,223 & 61,398 \\
\hline Varianza & & \\
Error & 3.29 & 1.00 \\
\hline
\end{tabular}

[Fuente] INEI - ENAHO(Encuesta Nacional de Hogares). Elaboración propia.
Tabla 5. Probabilidad de ocurrencia del evento (Odds Ratio)

\begin{tabular}{lccc}
\hline & Odds Ratio & Desv. Est. & Pr. Ocurr. \\
\hline PMgC & .08 & .002 & 11.96 \\
Vivienda & 1.13 & .009 & .13 \\
Agua Potable & .40 & .009 & 2.51 \\
Electricidad & .67 & .034 & 1.49 \\
Teléfono & 1.11 & .040 & 1.11 \\
Cable & 1.28 & .029 & 1.28 \\
Internet & 1.64 & .044 & 1.65 \\
Relac. Jefe de Hogar & 1.06 & .006 & 1.06 \\
Afiliación a la AFP & 1.66 & .051 & 1.66 \\
\hline [Fuente] INEI - ENAHO (Encuesta Nacional de Hogares). Elaboración propia.
\end{tabular}

[Fuente] INEI - ENAHO (Encuesta Nacional de Hogares). Elaboración propia. 